\title{
Thermal hysteresis of interface biased dipolar coupled nanoelements
}

Ana L. Dantas, Idalmir Queiroz, Gustavo Reboucas, Maria G. Dias, and A. S. Carriço

Citation: Journal of Applied Physics 113, 17D710 (2013);

View online: https://doi.org/10.1063/1.4795868

View Table of Contents: http://aip.scitation.org/toc/jap/113/17

Published by the American Institute of Physics

\section{Articles you may be interested in}

Thermal hysteresis of interface biased ferromagnetic dots

Journal of Applied Physics 102, 123907 (2007); 10.1063/1.2827478

Controlling the vortex core of thin Permalloy nano-cylinders dipolar coupled to Co polarizers Journal of Applied Physics 115, 17D110 (2014); 10.1063/1.4860056

Controlling the core-to-core distance of vortex pairs in exchange-biased iron elliptical nanoelements Journal of Applied Physics 111, 07D102 (2012); 10.1063/1.3670978

\section{Scilight}

Sharp, quick summaries illuminating the latest physics research

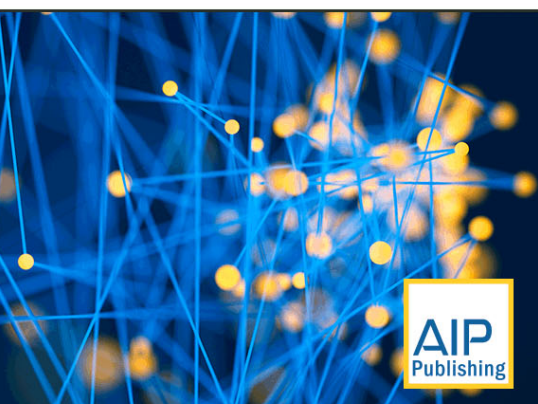




\title{
Thermal hysteresis of interface biased dipolar coupled nanoelements
}

\author{
Ana L. Dantas, ${ }^{1}$ Idalmir Queiroz, Jr., ${ }^{2}$ Gustavo Reboucas, ${ }^{2}$ Maria G. Dias, ${ }^{3}$ \\ and A. S. Carriço,a) \\ ${ }^{1}$ Departamento de Física, Universidade do Estado do Rio Grande do Norte, Mossoró, RN 59610-210, Brazil \\ ${ }^{2}$ Departamento de Física, Universidade Federal Rural do Semi-Arido, Mossoró, RN 59000-970, Brazil \\ ${ }^{3}$ Departamento de Física, Universidade Federal do Rio Grande do Norte, Natal, RN 59072-970, Brazil
}

(Presented 15 January 2013; received 1 November 2012; accepted 17 December 2012; published online 27 March 2013)

\begin{abstract}
We report a theoretical investigation of thermal hysteresis of a pair of interface biased elliptical iron nanoelements, separated by an ultrathin layer of nonmagnetic material. The thermal hysteresis originates in the strong dipolar interaction, and is tunable by the nature of the low temperature state and the eccentricity of the nanoelements. The width of the thermal hysteresis varies from $500 \mathrm{~K}$ to $100 \mathrm{~K}$ for lateral dimensions of $125 \mathrm{~nm} \times 65 \mathrm{~nm}$ and $145 \mathrm{~nm} \times 65 \mathrm{~nm}$. (ㅇ 2013 American Institute of Physics. [http://dx.doi.org/10.1063/1.4795868]
\end{abstract}

Thermal hysteresis has recently been investigated in alloys, ${ }^{1}$ multilayers, ${ }^{2}$ thin rare-earth films, ${ }^{3}$ and interface biased iron nanoelements. ${ }^{4}$

The thermal hysteresis of interface biased Fe nanoelements ${ }^{4}$ occurs at room temperature, well below the Fe Curie temperature. It originates in the thermal reduction of the interface field and the dipolar field rearrangements of the magnetic structure at high temperatures.

In this paper, we present a theoretical discussion of the thermal hysteresis of a pair of interface biased elliptical iron nanoelements separated by an ultrathin nonmagnetic insulating layer. The system consists of a pair of elliptical iron nanoelements $\left(\mathrm{NE}_{1}\right.$ and $\left.\mathrm{NE}_{2}\right)$, one of which $\left(\mathrm{NE}_{1}\right)$ is exchange coupled to an antiferromagnetic uncompensated substrate, as shown in the inset of Fig. 2. The exchange energy coupling spins at the interface layer of the iron nanoelement $\left(\mathrm{NE}_{1}\right.$ in Fig. 2) to spins of the antiferromagnetic substrate are represented by the interface exchange field, acting on the interface spins of $\mathrm{NE}_{1}$.

The thermal hysteresis of these systems originates in the fact that by heating to temperatures close to the Neel temperature of the substrate, there is a decay of the interface exchange field strength, allowing adjustments of the magnetic state of the nanoelements to suit the trends imposed by the strong dipolar interaction. The low temperature state turns unstable at some temperature close to the Neel point, and the system switches to another phase controlled by the intrinsic properties of the pair of nanoelements and the dipolar interaction between them. If the dipolar interaction is strong enough to compete with the interface exchange energy, the high temperature state may keep stable until low temperatures, during cooling. The sequence of states in the cooling branch may be different from that in the heating branch.

We focus on two special states at low temperatures. The parallel state (PS), with the magnetization of the nanoelements nearly parallel to each other, along the interface exchange field direction and the antiparallel state (AS), with the magnetization of the interface biased nanoelement

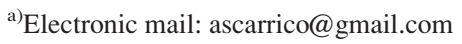

$\left(\mathrm{NE}_{1}\right)$, along the interface field direction and opposite to that of the second nanoelement $\left(\mathrm{NE}_{2}\right)$.

Around room temperature, the thermal stability of a pair of high eccentricity iron nanoelements tends to be large and relies almost entirely on the nanoelements dimensions. Also for separation of the order of $1 \mathrm{~nm}$, as currently used in the memory cells of spin transfer torque magnetic tunnel junctions (MTJs), the mutual dipolar interaction requires the nanoelements magnetizations in opposite direction. Therefore, we may anticipate much larger thermal effects upon heating the PS.

We consider three pairs of $10 \mathrm{~nm}$ thick iron nanoelements with lateral dimensions of $145 \mathrm{~nm} \times 65 \mathrm{~nm}, 135 \mathrm{~nm} \times 65 \mathrm{~nm}$, and $125 \mathrm{~nm} \times 65 \mathrm{~nm}$. The thickness of the nonmagnetic layer is close to $1 \mathrm{~nm}$ and one of the iron nanoelements is exchange coupled to a noncompensated $\mathrm{NiO}$ substrate.

We calculate the equilibrium magnetic states, starting at low temperature and going up to a temperature larger than the Neel temperature of the $\mathrm{NiO}$ substrate, and then proceeding back to low temperatures along the cooling branch.

We find that for any temperature the AS is strongly stabilized by the dipolar interaction. As shown in Fig. 1, the dipolar field strength within both nanoelements is around $7.5 \mathrm{kO}$ and is oriented parallel to the magnetization. As a result, the magnetic pattern in both nanoelements does not change along the heating branch. Therefore, the AS displays no thermal hysteresis. Thus, we focus on the thermal effects in the PS.
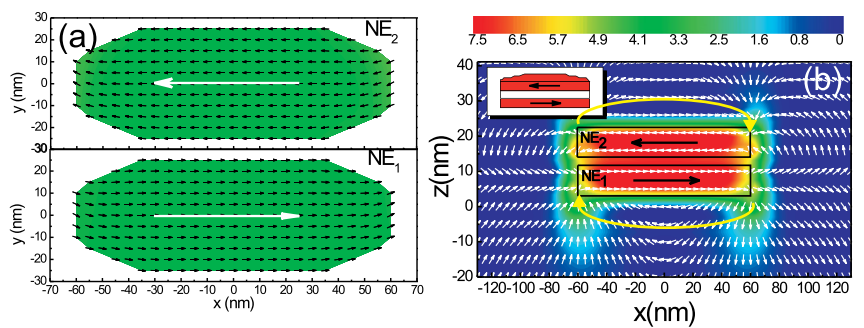

FIG. 1. (a) Magnetization pattern and (b) dipolar field, in the $y=0$ plane, of a pair of $10 \mathrm{~nm}$ thick iron elliptical nanoelements with lateral dimensions of $135 \mathrm{~nm} \times 65 \mathrm{~nm}$, separated by $1.1 \mathrm{~nm}$, and $H_{\text {int }}=1.18 \mathrm{kOe}$ in the antiparallel state. The bar code shows the strength of the dipolar field. 
The normal to the surface is chosen along the z-axis and the uniaxial anisotropy easy axis and the interface exchange field are both in-plane and along the $x$-axis. The antiferromagnetic substrate is held frozen in the antiferromagnetic order. We use cubic simulation cells with edge $d=5 \mathrm{~nm}$ and the energy density is given by

$$
\begin{aligned}
E= & \frac{A}{d^{2}} \sum_{j} \sum_{k}\left(1-\hat{m}_{j} \cdot \hat{m}_{k}\right)-M_{S} \vec{H}_{i n t} \cdot \sum_{i} \hat{m}_{i}-K \sum_{j}\left(m_{j}^{x}\right)^{2} \\
& +\frac{M_{S}^{2}}{2} \sum_{j} \sum_{k}\left(\frac{\hat{m}_{j} \cdot \hat{m}_{k}}{n_{j k}^{3}}-\frac{3\left(\hat{m}_{j} \cdot \hat{n}_{j k}\right)\left(\hat{m}_{k} \cdot \hat{n}_{j k}\right)}{n_{j k}^{5}}\right)
\end{aligned}
$$

where the first term is the intrinsic exchange energy coupling magnetic moments of nearest-neighbor cells and $A$ is the ferromagnetic exchange stiffness. The second term is the interface exchange energy, and the sum is restricted to the first layer of cells of $\mathrm{NE}_{1}$, at the interface. The third term is the anisotropy energy and the last term is the magnetostatic energy. $M_{S}$ is the saturation magnetization, $\hat{m}_{i}$ is the direction of the magnetic moment of the $i$-th cell, and $n_{i j}$ is the distance between the cells $i$ and $j$ in units of cell size $d$. We use $M_{S}=1.7 \times 10^{6} \mathrm{~A} / \mathrm{m}, A=2.5 \times 10^{-11} \mathrm{~J} / \mathrm{m}$, and $K=4.7$ $\times 10^{-4} \mathrm{~J} / \mathrm{m}^{3}$.

$H_{\text {int }}=J_{\text {int }}\left\langle S_{A F}\right\rangle / g \mu_{B} n_{\perp}$ is the magnitude of the interface exchange field, $\left\langle S_{A F}\right\rangle$ is the thermal average value of the AF interface spins, $n_{\perp}$ is the number of ferromagnetic atomic layers within a simulation cell, and $J_{i n t}$ is the interface exchange energy. For low temperature, we chose $H_{\text {int }}$ of the order of the intrinsic iron exchange field coupling neighboring cells (5.88 kOe).

$\mathrm{NiO}$ has a Neel temperature $T_{N}=525 \mathrm{~K}$ and is frequently used for biasing purposes. ${ }^{5}\left\langle S_{A F}\right\rangle$ has been calculated using an $S=1$ Brillouin function and full coordination of bulk-like $\mathrm{NiO}$ spins.

At low temperatures, the magnetic variables are initialized either close to the parallel state or to the antiparallel state. In order to simulate the thermal hysteresis process, for each temperature the magnetic variables are initialized near

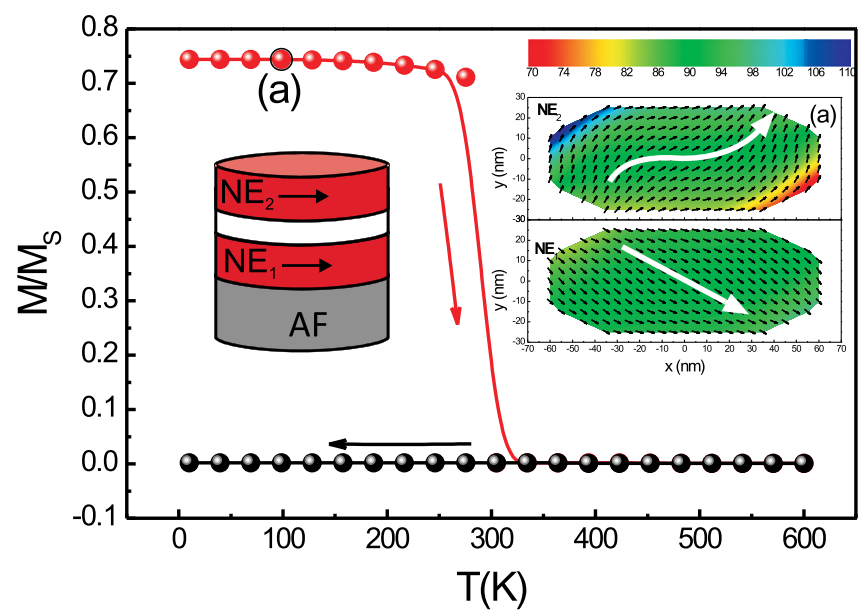

FIG. 2. Thermal hysteresis of a pair of $10 \mathrm{~nm}$ thick iron elliptical nanoelements with lateral dimensions of $135 \mathrm{~nm} \times 65 \mathrm{~nm}$, separated by $1.1 \mathrm{~nm}$, and $H_{\text {int }}=1.18 \mathrm{kOe}$. The inset shows the magnetization profile at low temperature. The bar code shows the angle with the $z$-axis. the equilibrium configuration of the previous temperature value. For each temperature, the equilibrium configuration is found by seeking a set of directions of the moments in all cells $\left(\hat{m}_{i}, i=1, \ldots, N\right)$ which makes the torque in each one of them smaller than $10^{-26} \mathrm{~J}$.

In Fig. 1, we show the dipolar field map in the xz-plane containing the major axis of a pair of $135 \mathrm{~nm} \times 65 \mathrm{~nm}$ $\times 10 \mathrm{~nm}$ nanoelements in the antiparallel state. The dipolar field is parallel to the magnetization and the intensity reaches $7.5 \mathrm{kOe}$ within both nanoelements. Due to the strong dipolar coupling, the antiparallel state is not affected by the strength of the interface exchange field. As a result, starting at low temperature in the antiparallel state there is no thermal hysteresis.

The thermal stability of the parallel state relies to a large extent on the interface exchange energy. There is a value of temperature above which the interface exchange field is no longer enough to hold the low temperature state stable. At this point, the magnetic phase is controlled by the dipolar field. The effective demagnetization anisotropy may be strong enough to stabilize the high temperature phase down to low temperatures in the cooling branch of the thermal loop. Three typical situations are shown in Figs. 2-4.

In Fig. 2, we show the magnetization of a pair of $135 \mathrm{~nm} \times 65 \mathrm{~nm}$ nanoelements separated by $1.1 \mathrm{~nm}$, for a low temperature interface exchange field strength of $1.18 \mathrm{kOe}$. The low temperature state $(T=100 \mathrm{~K})$ is shown in the inset, and consists of a pair of nearly parallel S-states. Both nanoelements have the spins oriented close to the interface exchange field direction, except for small areas near the ends along the major axis. This low temperature phase is kept with minor changes up to $\mathrm{T}=275 \mathrm{~K}$ where a transition to an antiparallel state occurs. There is no further changes until $\mathrm{T}=600 \mathrm{~K}$. This high temperature antiparallel state is strongly stabilized by the dipolar interaction between the nanoelements, and remains stable, in the cooling branch, down to low temperatures.

In Fig. 3, we show the magnetization of a pair of $125 \mathrm{~nm} \times 65 \mathrm{~nm}$ nanoelements separated by $1.1 \mathrm{~nm}$, for a

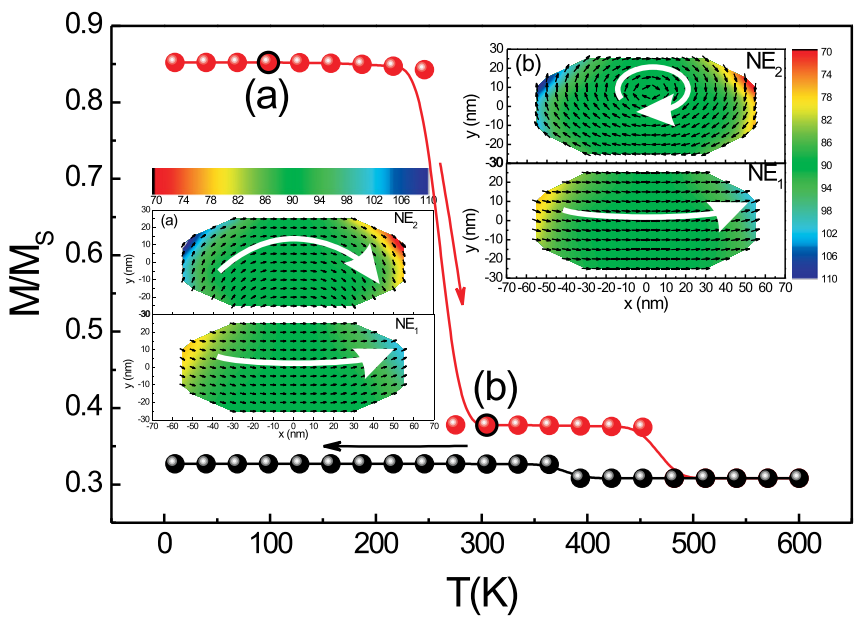

FIG. 3. Thermal hysteresis of a pair of $10 \mathrm{~nm}$ thick iron elliptical nanoelements with lateral dimensions of $125 \mathrm{~nm} \times 65 \mathrm{~nm}$, separated by $1.1 \mathrm{~nm}$, and $H_{\text {int }}=5.88 \mathrm{kOe}$. The inset shows the magnetization profile at selected points in the heating curve. The bar codes show the angle with the $\mathrm{z}$-axis. 

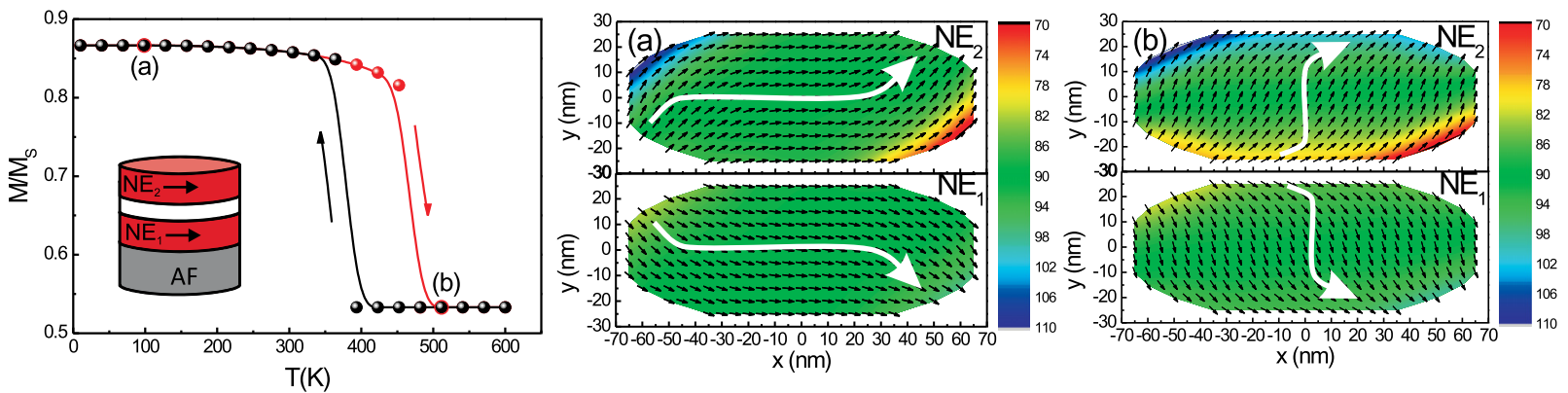

FIG. 4. Thermal hysteresis of a pair of $10 \mathrm{~nm}$ thick iron elliptical nanoelements with lateral dimensions of $145 \mathrm{~nm} \times 65 \mathrm{~nm}$, separated by $1.0 \mathrm{~nm}$, and $H_{\text {int }}=$ $1.18 \mathrm{kOe}$. The panels show the magnetization profile at selected points of thermal loop. The bar codes show the angle with the $\mathrm{z}$-axis.

low temperature interface exchange field strength of 5.88 kOe. The low temperature state $(T=100 \mathrm{~K})$ consists of a pair of nearly parallel buckle-states, as shown in the inset (a), with a parallel arrangement of the spins of the nanoelements, except for a small fraction near the ends along the major axis. This low temperature phase is kept with minor changes up to $T=249 \mathrm{~K}$ when the magnetization is reduced to $50 \%$ of the low temperature value due to nucleation of a vortex centered around the middle of the $\mathrm{NE}_{2}$, as shown in the inset (b). By further increasing the temperature, there is another small change in the magnetization due to vortex motion. Apart from minor changes in the vortex position, the high temperature vortex state remains stable, in the cooling branch, down to low temperatures.

In Fig. 4, we show the magnetization for a pair of $145 \mathrm{~nm} \times 65 \mathrm{~nm}$ nanoelements separated by $1.0 \mathrm{~nm}$, for a low temperature interface exchange field strength of $1.18 \mathrm{kOe}$. The low temperature state $(T=100 \mathrm{~K})$ consists of a pair of nearly parallel S-states, with a parallel arrangement of the spins of the nanoelements, except for a small fraction near the ends along the major axis. As the temperature increases, the magnetization of both nanoelements rotates (in opposite directions), eventually reaching an antiparallel perpendicular state, shown in the panel (b), at $T=500 \mathrm{~K}$. There is no further change up to $T=600 \mathrm{~K}$. Upon cooling the perpendicular state remains stable down to $T=400 \mathrm{~K}$, when a transition back to the low temperature state occurs. Notice that in this case the thermal hysteresis loop does not affect the room temperature state.

In conclusion, we have shown that interface biased pairs of elliptical iron nanoelements exhibit room temperature thermal hysteresis. The width of the thermal hysteresis, as well as the magnetization change in the thermal loop, is tunable by the dimensions of the nanoelements and the interface exchange field strength. We note that the present results may be valuable for the design of MTJ memory cells. ${ }^{6}$

The authors acknowledge financial support from FAPERN, CNPq, and CAPES.

${ }^{1}$ S. Demirtas, R. E. Camley, and A. R. Koymen, Appl. Phys. Lett. 87, 202111 (2005).

${ }^{2}$ J. P. Andrs, J. A. Gonzlez, T. P. A. Hase, B. K. Tanner, and J. M. Riveiro, Phys. Rev. B 77, 144407 (2008).

${ }^{3}$ A. L. Dantas, R. E. Camley, and A. S. Carriço, IEEE Trans. Magn. 42, 2942 (2006).

${ }^{4}$ A. L. Dantas, A. S. W. T. Silva, G. O. G. Rebouas, A. S. Carriço, and R. E. Camley, J. Appl. Phys. 102, 123907 (2007).

${ }^{5}$ P. A. A. van der Heijden et al., Appl. Phys. Lett. 72, 492 (1998).

${ }^{6}$ B. Dieny et al., Int. J. Nanotechnol. 7, 591 (2010). 